\title{
El paciente virtual como método de enseñanza en el pregrado
}

\author{
José Luis D'Addino, Horacio Mayorga, M. Ángeles Harris
}

Objetivos. Presentar un programa de trabajo práctico virtual para cirugía utilizando el correo electrónico y a través del aprendizaje basado en problemas, y evaluar la respuesta del alumnado.

Sujetos y métodos. Análisis retrospectivo y lineal. Se consideran los alumnos de los últimos cinco años, con un promedio de 18 alumnos por año. Total: 90 alumnos. Con cada uno de los tutores intervinientes en el proyecto se establecieron pautas de manejo y seguimiento de cada caso clínico quirúrgico que se daba a cada alumno. Todos los alumnos tenían un caso diferente entre sí que se repitió anualmente en cada curso. Algunos tutores seguían un solo caso y otros, dos. Cada alumno estableció con su tutor la manera más fácil y práctica para comunicarse, sea por teléfono, correo electrónico o personalmente. El tutor debía incentivar la búsqueda de información y hacer que el alumno se sintiera responsable del paciente. Cada caso, previamente establecido, debía ser operado, sufrir complicaciones, algunos fueron dados de alta y otros fallecieron. En base al número de alumnos cursantes, se determinaron los casos, siempre buscando que cada tutor desarrollara casos afines a las clases que daba o a su subespecialidad.

Resultados. El trabajo práctico virtual usando Internet fue muy útil para los estudiantes y benefició la relación docentealumno. La encuesta realizada a los alumnos mostró un $100 \%$ de satisfacción con el proyecto, lo mismo que la encuesta a los tutores.

Conclusión. La iniciativa de realizar un práctico con un paciente virtual fue sumamente satisfactoria para alumnos y tutores. Palabras clave. Paciente virtual.

\section{The virtual patient as a teaching method in undergraduate}

Aims. To present a virtual program about a practical work in Surgery using the web through problem based learning and to evaluate the student opinion.

Subjects and methods. Retrospective and lineal analysis. We included all the students in the last five years, with average of 18 students each one. Total: 90 pupils. Were established with each of the tutors, management guidelines and monitoring of each surgical case report that was given every student. All the students had a different case, repeated annually in each course. Some tutors had only one case and others two. Each pupil agrees with the tutor the easiest way to have a communication between them, such as phone, e-mail o in person. The tutor had to encourage the information research and make the student responsible of the patient. Every one of the cases, previously set, should have complications, some were extern and others died. Based on the number of students in each course, we decided the cases, always trying to find out cases according with the classes taught by the tutor and his specialization.

Results. The virtual program about a practical work in surgery has been very useful for the pupils and improved the relation between them and tutors. The search with students showed a $100 \%$ of satisfaction with this project, the same as with the tutors search.

Conclusion. The initiative of a working with a virtual patient has been extremely successful for both students and tutors.

Key words. Virtual patient.

\section{Introducción}

La práctica de los alumnos con pacientes es dificultosa y no siempre se cuenta con la suficiente cantidad de pacientes o patologías oportunas para lo que se enseña. El contacto temprano con la práctica clínica y la metodología de enseñanza basada en pro- blemas son estrategias que facilitan dicho aprendizaje. Nuestro objetivo es proveer casos clínicos virtuales de consulta electiva programada o de urgencia a cada alumno que cursa la asignatura de cirugía general, con un desarrollo individualizado para cada uno de ellos que será seguido de forma personalizada. Se crea de esta forma, a través del caso vir-
Servicio de Cirugía General (J.L. D'Addino, H. Mayorga); Departamento de Docencia (M.A. Harris). Hospital Municipal Vicente López. Facultad de Medicina. Universidad de Buenos Aires. Buenos Aires, Argentina.

Correspondencia: Dr. José Luis D'Addino. Adolfo Alsina 1991. Florida (1602). Buenos Aires, Argentina.

E-mail:

jldaddino@gmail.com

Agradecimientos:

A los tutores, Dres. Balmaceda Bresciani, Caubet, Duro, Geromin, Grosso, Herrera, Janeiro, Kalaydjian, Mendoza, Pigni, Quiroga, Rodríguez, Totino, Zanoni y Veira, que han colaborado desinteresadamente en este proyecto.

Conflicto de intereses: No declarado.

Competing interests: None declared.

(C) 2015 FEM 
tual y del vínculo electrónico, una estrecha relación alumno-docente que facilita la ejercitación cognitiva, de casos en tiempo real, pero sobre situaciones virtuales. Está fundamentado en el uso intensivo del correo electrónico y la búsqueda de información médica en Internet, bibliografía o consultas personales. La introducción de herramientas virtuales habilita una vía de escape a la constricción de los horarios hospitalarios aportando flexibilidad y extendiendo las posibilidades de colaboración y contacto entre docentes y alumnos.

\section{Sujetos y métodos}

Previo acuerdo entre todos los médicos docentes de cirugía general participantes del programa para el curso de la materia de cirugía general, se establecieron las pautas a seguir, basados en la técnica de SIPES y lo descrito por Hardin, considerando un modelo de 'aprendizaje basado en problemas' y teniendo en cuenta los pasos de la programación de la enseñanza. Se determinaron los mecanismos de comunicación entre los docentes y alumnos, en especial por correo electrónico, la evaluación continua a seguir, la forma de calificación final y la duración global del programa, con tiempo mínimo de dos semanas y máximo de seis, dentro de las cuales los pacientes virtuales pueden ser operados y dados de alta, ser reinternados por complicaciones o requerir nuevas intervenciones de acuerdo a cada patología preestablecida de antemano con cada tutor. Se determinaron las distintas patologías según el número de alumnos y de docentes intervinientes, incluyendo casos programados o casos de guardia, estableciendo con el tutor a cargo del caso qué complicaciones sufriría cada paciente en particular. Se pautaron casos para ser dados de alta, otros debieron ser reinternados, requerir reoperaciones o incluso fallecer. Nos basamos en las patologías genéricas más comunes para elegir los temas y siempre fue uno distinto por alumno. Todos los temas establecidos se repitieron anualmente manteniendo la evolución preestablecida de cada paciente virtual. Los pacientes se manejan según los algoritmos de tratamiento y metodología de estudios que se llevan a cabo en el Servicio de Cirugía de nuestro hospital, que están establecidos por escrito y se basan en la bibliografía internacional. Según el número de estudiantes, los casos de pacientes virtuales se reducían o aumentaban agregando complicaciones en la evolución del caso para mantener la relación de un caso por alumno, pero siempre con las mismas premisas y fundamentos para mantener una uniformidad en todos los casos y en todos los cursos anuales. Los antecedentes relacionados con la patología, así como los parámetros necesarios para confeccionar el identikit de cada paciente, fueron proporcionados por el tutor personal de cada alumno. La mayoría de los tutores tenía un solo alumno asignado, y en ocasiones tuvieron dos. Algunas veces se sumaron médicos residentes del último año como tutores. El primer día de clase, cuando se entregó a los alumnos el organigrama del curso con las clases y la concurrencia a consultorios y quirófano, se les facilitó una lista con los diferentes casos y el tutor a cargo. Los alumnos escogen el caso que desean y contactan con el tutor; al mismo tiempo, entre ellos deciden la manera de comunicarse para el seguimiento del paciente teniendo en cuenta que debe ser en tiempo real, con indicaciones, pedidos de estudios y recepción de resultados, interconsultas a otros especialistas, confección de una historia escrita con protocolo operatorio, etc. Cada alumno comienza cuando lo desea y lo finaliza según las pautas convenidas con el tutor. Elabora individualmente su trabajo pudiendo utilizar libremente todos los recursos disponibles, incluyendo consultas dentro o fuera del hospital, libros, revistas médicas, búsquedas bibliográficas o lo que crea necesario para llevar adelante su caso personal.

Se distribuyeron a los alumnos los 18 temas establecidos para los tutores intervinientes en el programa y se les explicó la evolución que se suscitaría en cada caso:

- Litiasis vesicular programada, que luego presentaría una litiasis coledociona residual y requeriría una colangiopancreatografía retrógrada endoscópica con papilotomía, buena evolución y alta; otro caso evolucionaría luego de la cirugía con una lesión de la vía biliar y se requeriría reoperación, anastomosis biliodigestiva, buena evolución y alta.

- Dolor abdominal agudo, que ingresó en urgencias y tenía antecedentes de litiasis vesicular, por lo que desarrollaría una pancreatitis aguda, se operaría de litiasis vesicular tras mejorar su cuadro agudo, buena evolución y alta.

- Eventración gigante programada, que dados los antecedentes del paciente (obesidad, diabetes, enfermedad pulmonar obstructiva crónica), requeriría neumoperitoneo y colocación de malla, buena evolución y alta.

- Hernia atascada, que sería operada de urgencias y se encontraría una lesión isquémica del intestino delgado, por lo que se efectuaría una resección y anastomosis. Requeriría alimentación enteral tamporaria por intestino corto, buena evolución y alta. 
- Síndrome coledociano, un caso se debería a panlitiasis coledociana que se operaría para una cirugía de anastomosis biliodigestiva, buena evolución y alta, y otro caso se debería a cáncer de cabeza de páncreas, que sería sometido a una pancreatectomía corporocaudal y se complicaría con fistula pancreática, sepsis y fallecimiento.

- Colecistitis aguda litiásica, que ingresaría en urgencias y se operaría, y posteriormente presentaría una infección del puerto umbilical, buena evolución y alta.

- Suboclusión intestinal, que llegaría a urgencias con gran distensión; antecedentes de múltiples laparotomías previas, se efectuaría laparotomía y se constatarían bridas, se resecaría el intestino delgado y presentaría luego una fístula que requeriría nutrición parenteral total, buena evolución y alta.

- Hemorragia digestiva baja, un caso se debería a una diverticulosis colónica que habría de intervenirse quirúrgicamente, buena evolución y alta, y otro caso sería por un cáncer de colon que sería sometido a una operación de Dixon, buena evolución y alta.

- Hemorragia digestiva alta, caso no descompensado a quien se diagnosticaría una úlcera gástrica sangrante, luego resangraría y debería ser operado, buena evolución y alta.

- Disfagia, dada por un cáncer de esófago del tercio inferior, que se sometería a una esofaguectomía sin toracotomía, buena evolución y alta.

- Bocio nodular eutiroideo, con punción con aguja fina que informaba Bethesda IV, cuya congelación resultaría un carcinoma papilar típico de tiroides, de $2 \times 3 \mathrm{~cm}$ de diámetro, luego desarrollaría una disfonía y tetania hipocalcémica, buena evolución y alta (Tabla I).

- Bocio hipertiroideo, que tras una tiroidectomía desarrollaría un hematoma sofocante con requerimiento de traqueostomía, buena evolución y alta.

- Traumatismo abdominal, por accidente de tráfico, se operaría y se produciría un estallido de bazo, presentaría una colección subfrénica, buena evolución y alta.

- Dolor abdominal de dos días de evolución, que se focalizaría en la fosa ilíaca derecha y se correspondería con una apendicitis aguda perforada con líquido intraabdominal, buena evolución y alta.

- Lesión cutánea en la pierna, se efectuaría resección de la lesión, cuya biopsia revelaría un melanoma Clark IV, Breslow de 4,0 $\mathrm{mm}$. Se efectuaría un estudio del ganglio centinela, vaciamiento ganglionar y evolucionaría mal, falleciendo el paciente por metástasis generalizadas.
Tabla I. Ejemplo de caso: nódulo tiroideo.

Paciente que consulta con un nódulo tiroideo

El alumno debe desarrollar, más allá de la anamnesis con los datos aportados por el tutor, los estudios iniciales

El tutor le responderá las preguntas y aportará resultados de los estudios que solicitó el estudiante El tutor hará énfasis en las preguntas de uso cotidiano que suelen hacer los pacientes y dudas diagnósticas que poseen para que el alumno pueda aclarárselas como si fuera a un paciente real

El alumno llegará al momento de la cirugía, lo operará y el tutor le responderá sobre la biopsia por congelación intraoperatoria para que determine la conducta quirúrgica a seguir

El estudiante deberá confeccionar la historia clínica, el parte operatorio y las indicaciones posquirúrgicas

El tutor revelará que el paciente presenta disfonía postoperatoria

El estudiante deberá tratar la complicación y evaluar estudios a solicitar

El Tutor informará que el paciente presenta tetania hipocalcémica

El alumno deberá medicar y evaluar la conducta a seguir con esta nueva complicación

El alumno externará al paciente en espera de la biopsia

El tutor le dará el resultado, que será un carcinoma papilar tiroideo con infiltración de la cápsula. El alumno deberá continuar el tratamiento de acuerdo a los criterios de tratamiento del cáncer diferenciado de tiroides

El alumno finalizará su caso cuando obtenga un centellograma con barrido corporal total negativo asociado a tiroglobulina y anticuerpos antitiroglobulina negativos

El tutor guiará al estudiante hasta este final

La historia escrita es evaluada y calificada de 1 a 10 por su tutor evaluando lo que el alumno realizó con su paciente durante toda la práctica

- Distensión abdominal de varios días de evolución, que ocurrió por una obstrucción colónica neoplásica en sigmoides, se realizaría una hemicolectomía izquierda, buena evolución y alta.

- Tumor de parótida de larga evolución, la punción con aguja fina resultaría sospechosa y la biopsia postoperatoria revelaría un carcinoma adenoidequístico, evolucionaría con una parálisis facial, buena evolución y alta.

- Abdomen peritoneal, que ingresaría en urgencias y tendría como antecedentes la ingesta de aspirinosímiles y en la operación se hallaría una úlcera gástrica perforada que se sometería a cierre simple, buena evolución y alta.

Se realizaron 90 historias clínicas reales de pacientes virtuales, basados en los 18 temas originales a razón de uno por alumno, que se repitieron en cada curso. Cuando se requerían más temas, se tomaron dos evoluciones distintas para un mismo caso en aquellas patologías más frecuentes, como litiasis vesicular o síndrome coledociano, y cuando debía reducirse el número, se excluyeron melanoma, tumor de parótida o disfagia, por considerarse de menor frecuencia en base a la casuística del Servicio 
Tabla II. Resultado de la encuesta anónima a los 90 alumnos participantes.

\begin{tabular}{lcccccccc}
\hline & 1 & 2 & 3 & 4 & 5 & $\mathrm{n} / \mathrm{c}$ & $(1+2) /$ total \\
\hline Le ha resultado útil el desarrollo del programa & 86 & 4 & 0 & 0 & 0 & 0 & 1 \\
\hline Lo motivó a estudiar y dedicar más tiempo al tema & 85 & 4 & 1 & 0 & 0 & 0 & 0,98 \\
\hline Contribuyó a estimular la búsqueda sobre el tema & 72 & 10 & 6 & 1 & & 1 & 0,91 \\
\hline Le ayudó a mejorar sus habilidades diagnósticas & 68 & 11 & 5 & 4 & 2 & 0 & 0,87 \\
\hline Le ayudó a confeccionar una historia clínica & 83 & 6 & 1 & 0 & 0 & 0 & 0,98 \\
\hline Le permitió aprender a hacer indicaciones & 87 & 2 & 0 & 0 & 0 & 1 & 0,98 \\
\hline Sintió que trataba un paciente real & 79 & 6 & 3 & 2 & 0 & 0 & 0,94 \\
\hline Recomendaría continuar este programa & 86 & 4 & 0 & 0 & 0 & 0 & 1 \\
\hline Sintió que se relacionaba mejor con su docente & 72 & 10 & 7 & 0 & 1 & 0 & 0,91 \\
\hline
\end{tabular}

de Cirugía General; esto se determinaba según el número de alumnos que remitía la Facultad de Medicina de la Universidad de Buenos Aires para cada una de los cursos. En promedio fueron 18 alumnos por año, con un mínimo de 15 y un máximo de 21 . El total considerado fue de 90 participantes, distribuidos entre 2006 y 2011.

El número de docentes varió según el plantel de alumnos. Regularmente, el Servicio de Cirugía General cuenta con 12 médicos dispuestos a ejercer la docencia. Ocasionalmente hemos sumado al jefe de residentes y a residentes de último año. Cada docente recibió una explicación del caso con su evolución final, y si se cambiaba el tutor, los criterios de la evolución del caso se respetaban según lo pautado para cada paciente virtual en cada curso anual. La función del tutor fue crear un hábito de búsqueda de información y de investigación continua en el estudiante, haciéndole sentir que el paciente estaba bajo su total responsabilidad de forma diaria y permanente, dependiendo del alumno la toma de decisiones y conductas. El tutor debe guiar y sugerir, pero no indicar, y ha de permitir al alumno equivocarse para luego, al finalizar el trabajo práctico, explicarle los errores. Fueron dados conceptos claves para que se aplicaran principios y técnicas basados en conocimientos supervisados por los docentes.

Los alumnos debieron confeccionar una historia clínica tomando como modelo las historias clínicas del Servicio de Cirugía General del hospital, lo que incluyó desarrollo de la historia, seguimiento hospitalario y ambulatorio, hoja de indicaciones, protocolo operatorio, pedidos de interconsultas, etc.
Al finalizar con los pacientes virtuales, cada alumno presentaba la historia a su tutor para la evaluación y devolución personalizada e individualizada. Se calificó la historia de 1 a 10.

Se pusieron en práctica encuestas para los alumnos, incluyendo una parte abierta a comentarios y una parte estructurada con 10 ítems a los que los participantes atribuyeron una escala de puntuación de 1 a 5 de acuerdo a una escala de tipo Likert (1: totalmente de acuerdo; 2 : en parte de acuerdo; $3:$ ni de acuerdo ni en desacuerdo; 4: en parte en desacuerdo; 5: totalmente en desacuerdo). Interpretamos como concordante o afirmativa una respuesta con porcentaje de 0,70 o mayor para la suma de las opciones 1 y 2 sobre el total de respuestas, y como discordante o negativa cuando el porcentaje fue de 0,30 o menor (Tabla II). Todos los cursos completaron la encuesta de forma anónima. También se encuestó de forma oral a los tutores sobre el caso y la motivación de los alumnos (Tabla III).

En un principio, la historia clínica de un paciente virtual la tomamos como un trabajo práctico que se consideraba como nota de concepto para el final, pero luego, con las encuestas, se cambió y en los últimos tres años se decidió que el prefinal fuera directamente el caso que habían investigado y tratado. De esta forma, en dicho examen, se les toma a los alumnos el caso y se hacen preguntas sobre el tema.

\section{Resultados}

La evaluación del programa que iniciamos en 2006 y continúa hasta la fecha fue realizada diariamente por el coordinador del programa mediante consultas a los alumnos y docentes y chequeo de la casilla de correo electrónico. El 90\% de los alumnos optaron por no usar la casilla general sino usar la personal y contactar directamente con el docente. En cinco años han realizado este programa de paciente virtual 90 alumnos, con una media de 18 por año. Se confeccionaron en total 90 historias clínicas reales de los pacientes virtuales, basados en los 18 temas originales. Ningún tema debió ser cambiado ni alterado durante el desarrollo del presente estudio. Realizamos encuestas anónimas a alumnos y docentes en cada curso. Evaluamos los resultados del programa de manera retrospectiva y lineal. El 100\% de los alumnos no solo desarrolló una historia clínica correcta, sino que asignó a sus pacientes datos de filiación y familiares más allá de lo esperado. El 98\% confeccionó desde el consentimiento informado hasta la dirección y el teléfono. La totalidad del alumnado consiguió radiografías de tórax y proto- 
colos de estudios afines a los solicitados para cada caso en particular, ya sea laboratorios, ecografías o incluso informes histopatológicos. Resultó llamativa la compenetración de cada estudiante con su paciente, al que asumieron con responsabilidad total. Hubo casos de alumnos que incluyeron interconsultas con otras especialidades, sobre todo cuando ocurrieron complicaciones en la distinta evolución de los casos. Dichas interconsultas fueron escritas por los especialistas a los que los alumnos específicamente buscaron. Los docentes se sintieron muy motivados por el requerimiento de los alumnos y el 95\% de los tutores manifestó que el alumno asignado les cuestionaba opiniones incluso remitiéndoles bibliografía para confrontar su opinión. En muchas oportunidades, cuestionaban los algoritmos establecidos para estudios o tratamientos y basaban sus diferencias de opinión en búsquedas en Internet o consultas con otros profesionales, lo que determinaba una fluida y permanente comunicación con el tutor. Nos asombró comprobar la adhesión al programa; algunos docentes que se tomaban días libres seguían conectados por correo electrónico con sus alumnos. En general, todos los tutores se refirieron a la actitud de sus alumnos como de 'asedio' durante el desarrollo del programa.

En todas los cursos en los que hemos puesto en práctica este tipo de trabajo práctico, el 98\% de los alumnos manifestó una total satisfacción, el 96\% expresó que les permitió aprender un tema en profundidad, el $100 \%$ agradeció que le enseñaran a hacer indicaciones, planes de hidratación, antibioticoterapia, etc, tareas no frecuentes de aprender en el pregrado. Hemos comprobado que, a diferencia de años anteriores, cuando no usábamos este tipo de trabajo práctico, esta herramienta motivó a los alumnos a realizar prácticas con pacientes ya sea en urgencias o en la consulta, donde se interesaban en colocar una sonda nasográstrica, vías periféricas, sondas vesicales, curación de heridas o extracción de suturas, etc. Las respuestas de las encuestas muestran un importante acuerdo de los alumnos participantes: les resultó útil (1), los motivó a estudiar y dedicarse más al tema $(0,98)$, contribuyó a estimular la búsqueda $(0,91)$, ayudó a mejorar habilidades diagnósticas $(0,87)$, les permitió confeccionar una historia clínica $(0,98)$, les enseño a hacer indicaciones $(0,98)$, sintieron que trataban a un paciente real $(0,94)$, recomendarían el programa (1) y se relacionaron mejor con el tutor $(0,91)$.

Todas las historias fueron calificadas según una observación sistemática, de forma continua, y al final, cada docente-tutor explicó personalmente los errores cometidos durante el seguimiento, la tera-
Tabla III. Resultado de la encuesta a 12 tutores.

\begin{tabular}{lccccccc}
\hline & 1 & 2 & 3 & 4 & 5 & $\mathrm{n} / \mathrm{c}$ & $(1+2) /$ total \\
\hline Le ha permitido incentivar el estudio de un tema & 12 & 0 & 0 & 0 & 0 & 0 & 1 \\
\hline Lo motivó a conectarse con el estudiante & 10 & 1 & 1 & 0 & 0 & 0 & 0,91 \\
\hline Sintió que el alumno se responsabilizaba del paciente & 9 & 2 & 1 & 0 & 0 & 0 & 0,91 \\
\hline Le agradó el programa & 12 & 0 & 0 & 0 & 0 & 0 & 1 \\
\hline Sería nuevamente tutor & 12 & 0 & 0 & 0 & 0 & 0 & 1 \\
\hline
\end{tabular}

péutica o la solicitud de estudios. Se consideró para la calificación del trabajo práctico la confección de la historia clínica (presentación y contenido), las evoluciones, las solicitudes de estudios o interconsultas, las indicaciones, la descripción del protocolo operatorio y el manejo postoperatorio. Para cada ítem se estableció una puntuación de 1 a 10 y del promedio quedaba la nota final y única del paciente virtual. El promedio de la calificación final de los pacientes virtuales fue de 8 puntos.

Las encuestas a los tutores también reflejaron un altísimo interés por este programa y satisfacción por él (1), manifestaron que serían nuevamente tutores (1), les permitió que el alumno estudiara profundamente un tema (1) y los alumnos sintieron que era un paciente real y se motivaron a conectarse mutuamente $(0,91)$.

Inicialmente el programa se había pensado como un simple trabajo práctico, pero luego los alumnos solicitaron que promediara con el examen final o se tomara como una prueba parcial. Observando la ardua labor que hacían los alumnos y en base a las propuestas de las encuestas, optamos por evaluar a los alumnos con dos parciales y utilizar el paciente virtual para el prefinal; de esta forma, los alumnos pueden explayarse sobre un tema sabiéndolo a fondo. Procuramos que el examinador no haya sido su tutor.

\section{Discusión}

La enseñanza del pregrado y la práctica directa con pacientes es sumamente dificultosa. Debe ser integral, donde la instrucción teórica, académica, docente, científica y de investigación, y también los aspectos ético-morales, sean tan importantes como el aprendizaje y el entrenamiento. Un principio fundamental que debe permanecer siempre es el compromiso con las necesidades de nuestros estudiantes, quienes serán nuestros sucesores y, paralela- 
mente, salvaguardar los mejores intereses de nuestros pacientes a quienes cuidamos.

Se acepta que se retiene el $20 \%$ de lo que se escucha, el $30 \%$ de lo que se ve y el $90 \%$ de lo que se ve, se escucha y se hace $[1,2]$. Esto es lo que buscamos con nuestro proyecto de práctica con paciente virtual.

Las encuestas de opinión de alumnos revelan reiteradamente que los trabajos prácticos son una de las actividades que más insatisfacción genera $[3,4]$. Varios estudios hacen referencia a que se identificaron estrategias para la formación de estudiantes que se resumen en seis alternativas [5]: centrada en el estudiante, basada en problemas, integrada, orientada a la comunidad, basada en cursos optativos, y sistemática.

En 1991, un análisis del Centro de Educación Médica de la Universidad de Dundee, Escocia, propuso un modelo curricular de enseñanza conocido como SPICES (student, problema, integrated, comunity, elective and sistematic) en el cual nos basamos para nuestro proyecto. La programación de la enseñanza se fundamenta en $[4,6]$ : propósito, destinatarios, objetivos, contenidos, estrategias, recursos y evaluación. El desarrollo lo realizamos siguiendo las pautas de la programación y adaptando una estrategia que comenzó a desarrollarse en 1960 en las escuelas de medicina de Norteamérica y Europa, el 'aprendizaje basado en problemas' [5,7]. En los últimos años ha surgido una tendencia mundial de dirigir la educación médica hacia un enfoque basado en las competencias $[1,8]$. La evaluación más eficiente debe hacerse de forma continua, con la observación de la ejecución, mediante control del desempeño, considerando los conocimientos adquiridos, actitudes, valores y habilidades demostradas, búsqueda de información, desarrollo y seguimiento del caso [1]. La utilización de múltiples instancias de evaluación, como los exámenes y la observación sistemática, acercan a la evaluación holística [7]. La enseñanza basada en problemas supone una disminución de la jerarquía y la dirección del docente, al tiempo que estimula el trabajo autónomo del alumno y exige que el profesor se desempeñe como animador y tutor del proceso de aprendizaje. El uso de un paciente virtual permite a los alumnos desarrollar una práctica completa desde el prequirúrgico hasta el alta, con automotivación tanto en la investigación bibliográfica como en la práctica manual. Así mismo, los acostumbra al uso de algoritmos y esquemas de tratamiento. Aun cuando les exige tiempo y esfuerzo, los alumnos de cirugía general perciben rápidamente que el programa desarrolla en ellos habilidades que les serán de real utilidad en la práctica médica; por otra parte, la tutoría electrónica resulta un estí- mulo y guía para que los estudiantes amplíen y profundicen los propios conocimientos a través de un proceso de investigación e intercambio [9]. El estudio de una materia que tiene un gran componente práctico es problemático con pacientes reales, tanto por los aspectos legales como por la disponibilidad de casos en el momento que cursan los estudiantes, pero utilizando una estructura virtual los alumnos sienten que están tratando un paciente real [1,2]. El análisis de la ejecución del programa del paciente virtual, asociado a la evaluación con encuestas anónimas, permitió la libre expresión del estudiante [10-12], la observación continua de las actividades docentes por parte del coordinador y la relación en conjunto, posibilitaron correlacionar lo planificado con lo ejecutado, estableciéndose un equilibrio 'producto-resultado' de gran aceptación.

En conclusión, esta experiencia ha demostrado que, utilizando una herramienta virtual, el alumno cumple con los objetivos planteados para el trabajo práctico en el curso de cirugía general y que este programa puede implementarse en donde la práctica directa con pacientes es dificultosa. Además, resultó muy gratificante tanto para los docentes como para los alumnos, permitiendo una relación interdisciplinaria estrecha e inédita y un incentivo de trabajo mutuo no visto anteriormente.

\section{Bibliografía}

1. Valenzuela $\mathrm{CH}$. Entrenamiento y evaluación del cirujano en formación. Parte I. Rev Arg Cirugía 2013; n. ${ }^{\circ}$ extr.: 1-34.

2. Venturrelli J, ed. Nuevos enfoques, metas y métodos. Washington: OPS; 1997.

3. De Ketele JM. Observar para educar. Madrid: Aprendizaje Visor; 1989.

4. Traynor O, Gruwez JA, Darzi A, Broos P, Kristoffel R, Dumon $\mathrm{K}$, et al. Educación quirúrgica en el nuevo milenio: perspectiva militar. Clínicas Quirúrgicas de Norteamérica 2004; 6: 1401-20.

5. Galli A. Programa de formación de formadores de ciencias de salud. Asociación Amigos de la Facultad de Medicina. Prensa Médica Argentina 2004; $1: 7$.

6. Schmidt HG. Problem based learning: rationale and description. Med Educ 1983; 17: 11-6.

7. Galli A. Evaluación de las competencias adquiridas por los estudiantes. Evaluación de la enseñanza. Módulo n. ${ }^{\circ} 3$. Buenos Aires: AFACIMERA; 2012.

8. Wassermann AO, Harris MA, Bernabó JG. Un sistema para hallar oportunidades de mejora en la enseñanza basado en la percepción de los alumnos. Educ Med 2006; 9: 66-74.

9. Bernabó J. Enseñanza de habilidades diagnósticas complejas a través de un programa integrado (B-learning) de enseñanza basada en problemas: experiencia de seis años. Revista Argentina de Educación Médica 2010; 4: 27-33.

10. McCormack L. Introducción de la simulación y la evaluación en la formación del cirujano del siglo XXI. Rev Arg Cirugía 2013; n. ${ }^{\circ}$ extr.: 39-86.

11. Canale H. Planificación de recursos humanos. Washington: OPS; 1989.

12. Brailovsky A. Educación médica, evaluación de las competencias. In OPS-OMS, eds. Aportes para un cambio curricular en Argentina. Buenos Aires: Universidad de Buenos Aires; 2001. 